\title{
Evaluación comparativa del sistema de control interno del sector comercial y del sector público del Cantón Morona
}

\section{Benchmarking of internal control system of commercial sector and public sector of Canton Morona}

\author{
López Jara, Ana Alexandra* \& Pesántez Rodríguez, Judith Cristina \\ Universidad Católica de Cuenca, Sede Macas, Ecuador \\ *alopezj@ucacue.edu.ec
}

\begin{abstract}
Resumen
El desarrollo de la economía y la tecnología en una sociedad, provoca que en las entidades públicas y privadas se convierta en un desafío la mitigación de los riesgos que puedan afectar la consecución de los objetivos en la organización. Un sistema de Control interno, constituye un proceso integral diseñado y efectuado por la máxima autoridad y todos los miembros de la organización, con el objetivo de: confiabilidad de la información financiera; dar cumplimiento a leyes y obligaciones; y la salvaguarda de los recursos o activos. Los Sistemas de Control Interno son diseñados de acuerdo a la naturaleza y operaciones de la entidad, sin embargo, existen modelos universales de Control Interno, siendo uno de estos el Sistema de Control COSO I. La investigación consistió en evaluar de forma comparativa a los Sistemas de Control Interno en el Sector Privado y en el Sector Público, del cantón Morona, aplicando como herramienta de evaluación el cuestionario según el modelo COSO I. Los resultados demuestran que en ambos sectores presentan un alto compromiso con la organización y hacia los objetivos que lideran su desarrollo, observándose que en el sector público los procedimientos se encuentran en función a bases legales, mientras que en el sector privado existe ausencia de formalismo al implementar estas prácticas.
\end{abstract}

Palabras clave: Sistema de Control Interno, Manual de Normas y Procedimientos, Modelo de Control Interno COSO.

\begin{abstract}
The development of economy and technology, in a society, causes in public and private entities, it becomes a challenge mitigating the risks that may affect the achievement of the objectives of the organization. A system of internal control, is a comprehensive process designed and made by the highest authority and all members of the organization, with the aim of: reliability of financial information; comply with laws and obligations; safeguard the resources or assets. The Internal Control Systems are designed according to the nature and operations of the entity, but there are universal models Internal Control, one of these Control System COSO I. The research was to evaluate comparatively the Internal Control Systems in the private sector and the public sector, the canton Morona, using as an evaluation tool the questionnaire according to the COSO model I. the results show that in both sectors have a high commitment to the organization and the objectives leading its development, however in the public sector they are procedures according to legal bases, while in the private sector there is an absence of formalism in implementing these practices.
\end{abstract}

Key words: Internal Control System, Standards and Procedures Manual, COSO Internal Control Model.

\section{Introducción}

Las organizaciones en orden con el desarrollo científico y tecnológico, han modificado sus estructuras en función a los cambios requeridos, entre ellos han centrado la atención al diseño de controles internos, que promuevan la eficiencia en la operaciones, la salvaguarda de activos y la reducción de los riesgos, garantizando fiabilidad de los estados financieros en observancia al cumplimiento de las leyes y normas vigentes.

Estos elementos son analizados como estrategias a la solución de problemas potenciales que afectarían la consecución de los objetivos.
Hasta los años setenta, en las áreas de diseño de sistemas y en auditoría predominaban las actividades de control. En el mismo orden que el desarrollo tecnológico primó en las organizaciones, los riesgos fueron ocupando el primer lugar, y es así que a partir de las investigaciones del caso Watergate, entre los años 1973 y 1976, se evidenciaron limitaciones significativas del control interno, que promovieron el desarrollo de actividades ilegales de financiación de grupos políticos, incluso sobornos a líderes de gobiernos extranjeros.

Desde entonces, el control interno en las organizaciones se convirtió en un tema de interés tanto para gobiernos, 
empresas privadas y organizaciones en general, y por tal motivo, se dictaron normas (Foreign Corrupt Practices Act, pronunciamientos de AICPA y SEC), se conformaron comisiones especiales de investigación de riesgos (Comisión Cohen, Commisión Treadway, Comité Minahan) y se efectuaron estudios prácticos en numerosas organizaciones.

Este crecimiento provocó cambios en las organizaciones tales como: responsabilidad de llevar registros, sistemas de control interno (CI) y obligación de emitir documentos respecto a la valoración de las actividades de control.

El control interno, desde su origen, no tenía el mismo significado en todo el mundo. Existía una variedad de contextualizaciones respecto al Control Interno. Es por ello, que en el año 1992, se publicó el Internal Control - Integrated Framework (COSO I), por el Committee of Sponsoring Organizations of the Treadway Commission, conocido como COSO.

Así se consolidaron múltiples conceptos sobre "CI" en un "Marco", que establece una definición general y componentes de control. Este documento fue considerado elemento básico en la valoración de los sistemas de "CI" de las organizaciones.

De lo presentado anteriormente, se analiza que es responsabilidad de todos los miembros de una organización la creación, el cumplimiento y fomento del control interno a fin de alcanzar una optimización de recursos en el tiempo adecuado, validez de la información financiera y fiel cumplimiento a las leyes y regulaciones.

La Contraloría General del Ecuador, mediante el acuerdo N 039 - CG - 2009, señala los lineamientos del marco contextual de Las Normas Técnicas de Control Interno para el Sector Público, N 100-01, donde se enfatiza la instrumentalización de manuales y procedimientos para las operaciones de una organización, a fin de fortalecer los controles integrados al sistema de control interno y promover fortalezas frente a la gestión.

En virtud de lo expuesto, la importancia del sistema de control interno para cualquier organización, radica en el diseño, implementación y funcionamiento de los procedimientos de control acorde a la naturaleza de las operaciones, los cuales corresponden al soporte para efectuar cambios necesarios bajo marcos de eficiencia, eficacia, efectividad, economía, ecología y ética en los procesos.

(Rivas Márquez, 2011), en el estudio sobre la evolución de los Sistemas de Control Interno, fundamentado en el aporte de (Mantilla Blanco, 2005), destaca que los modelos de control organizacional han sufrido modificaciones en la medida que se han transformado las estructuras empresariales y con ello ha evolucionado la auditoría. La siguiente tabla 1 elaborado a partir de la revisión bibliográfica presenta un resumen de las generaciones de control interno investigadas:

(y Lybrand, 1997), señalan que al existir una gran variedad de definiciones o entendimientos acerca del Control Interno, en el año 1992, el Committee of Sponsoring Organizations of the Treadway Commission, conocido como
Tabla 1

Resumen de generaciones de control interno

\begin{tabular}{|c|c|}
\hline \multicolumn{2}{|r|}{ Control Interno } \\
\hline Primera generación & $\begin{array}{l}\text { Esta etapa se caracterizó por el desarrollo de } \\
\text { procedimientos contables y administrativos, } \\
\text { fundamentados en acciones empiricas de ensayo y } \\
\text { error. Los controles diseñados por una carente } \\
\text { profesionalización de quienes tienen a su cargo el } \\
\text { sistema de control interno. }\end{array}$ \\
\hline Segunda generación & $\begin{array}{l}\text { Esta etapa se encuentra marcada por el sesgo legal. } \\
\text { Las entidades del sector público, tuvieron que } \\
\text { asumir estructuras y prácticas de control interno, de } \\
\text { cumplimiento formal, lejos de los niveles de calidad } \\
\text { y técnicos requeridos. El control interno constituye } \\
\text { un medio para definir el alcance las pruebas de } \\
\text { auditoria, siendo institucionalmente el plan de } \\
\text { organización, el conjunto de métodos y } \\
\text { procedimientos para asegurar que los activos están } \\
\text { debidamente protegidos, que los registros contables } \\
\text { son fidedignos y que las actividades de la entidad se } \\
\text { desarrollan eficazmente. }\end{array}$ \\
\hline Tercera generación & $\begin{array}{l}\text { En la última década los controles internos han } \\
\text { tomado una orientación dirigida de manera } \\
\text { prioritaria a fomentar la eficiencia, reduciendo el } \\
\text { riesgo en la consecución de los objetivos, ayudan a } \\
\text { asegurar la confiabilidad de la información } \\
\text { financiera y la de gestión, a proteger los recursos, y } \\
\text { a cumplir las leyes, reglamentos y cláusulas } \\
\text { contractuales aplicables. Es aqui donde se reconoce } \\
\text { el fruto de los esfuerzos originados por COSO hacia } \\
\text { los años } 90 \text { 's, los cuales han sido complementados } \\
\text { por los nuevos direccionamientos estratégicos, el } \\
\text { ascenso en la escala organizacional y, reforzados } \\
\text { por los alcances de la Sarbanes-Oxley Act de } 2002 \text {. }\end{array}$ \\
\hline
\end{tabular}

COSO, conformado por los cinco organismos profesionales de control más relevantes de los Estados Unidos, después de cinco años de investigación, análisis y discusión, se publicó el Internal Control - Integrated Framework (COSO I), presentándose un nuevo marco conceptual del control interno que integra las variadas definiciones vigentes sobre Control Interno, de la siguiente manera:

El control interno es un proceso, ejecutado por el consejo de directores, la administración y otro personal de la entidad, diseñado para proporcionar seguridad razonable con miras a la consecución de objetivos en las siguientes categorías:

1) Eficiencia y eficacia en las operaciones.

2) Confiabilidad de la información financiera.

3) Desempeño en observancia a las leyes y regulaciones

En conformidad al marco conceptual integrado del modelo de Control Interno COSO, este procedimiento presenta componentes que la alta gerencia elabora e implementa para promover una seguridad razonable en la consecución de sus objetivos de control en conformidad a los componentes que se detallan a continuación:

\subsection{Componentes del modelo de Control Interno COSO}

Los componentes son: (a) Entorno de Control, (b) Evaluación de los Riesgos, (c) Actividades de Control, (d) Información y comunicación, y (e) Supervisión. En 
detalle se presenta la caracterización de cada componente del modelo de control interno COSO.

\subsubsection{Entorno de Control}

El entorno de control tiene una influencia profunda en la manera como se estructuran las actividades del negocio, se fijan los objetivos y se consideran los riegos.

Este componente se ve dirigido por la cultura de la organización, es decir, políticas, procedimientos, códigos de ética, que fomenten la conciencia de control de los miembros de la entidad. Es en este componente donde se generan las actividades de las organizaciones, las cuales responden a la filosofía de gestión de la gerencia, compuesto por el compromiso ético de los accionistas y la integridad que debe reflejarse en todos los niveles de la entidad. A partir de la misión y visión de la organización, surge el compromiso de los miembros a desarrollar sus operaciones alineado a las directrices de la alta gerencia.

Los factores que integran este componente comprenden: la integridad y los valores éticos, los incentivos para el personal, el compromiso para la competencia, la filosofía y estilo de operación de la administración, la estructura organizacional, el consejo de directores o comité de auditoría, la asignación de autoridad y responsabilidad, las políticas y prácticas sobre recursos humanos, entre otros.

Para lograr un ambiente de control adecuado se deben tomar en cuenta aspectos como: el estilo de gerencia, la estructura organizacional, el compromiso por ser mejores y la división del trabajo (Pany, 2005).

En concordancia a lo expuesto se entiende que el ambiente de control crea el tono de la organización al influir en la conciencia de control del personal y puede verse como el fundamento de los demás componentes.

\subsubsection{Evaluación de los Riesgos}

La entidad debe conocer, predecir y aproximarse a los riesgos, que podrían afectar la consecución de los objetivos, con el objeto de implementar mecanismos que identifiquen, examinen y mitiguen los potenciales riegos.

Se pueden diferenciar dos tipos de riesgos en una organización: el riesgo de control y el riesgo inherente. El riego inherente es propio de las actividades o procesos de la organización, que pueden incidir en la probabilidad de continuar el postulado de negocio en marcha; en tanto que el riesgo de control se entiende como la posibilidad de que presentándose errores de importancia no puedan ser detectados por los sistemas de control de la entidad impidiendo el logro de los objetivos organizacionales (Viloria, 2005).

Existen metodologías favorables para la identificación y valoración de los riesgos, entre las más comunes encontramos las matrices y los manuales de referencia. En este proceso de identifican riesgos interno y/o externos, por cada objetivo importante a nivel de actividad. Posterior a la identificación y valoración es necesario reducir, administrar o compartir los riesgos, se puede realizar este análisis mediante un plan de mitigación.

\subsubsection{Actividades de control}

En la estructura organizacional se establecen procedimientos que ayudan a asegurar que las políticas y normas se lleven a cabo con una seguridad razonable, con la finalidad de mitigar los riesgos existentes que entorpecen la consecución de objetivos de la entidad. Se pueden mencionar como actividades de control más comunes a: el procesamiento de Información y el control de desempeño. (Viloria, 2005)

(Blanco Luna, 2012), caracteriza como acciones de las personas para implementar las políticas, asegurando el cumplimiento de las directrices administrativas identificadas como necesarias para administrar los riesgos. El autor presenta que las actividades de control deben realizarse en observancia a los objetivos de la entidad, los cuales se relaciona: operaciones, información financiera, o cumplimiento.

Entre los tipos de actividades de control se pueden encontrar: revisiones de alto nivel, controles físicos, procesamiento de información, indicadores de desempeño, segregación de responsabilidades, entre otros.

\subsubsection{Información y comunicación}

La información es necesaria en todos los niveles de la organización para operar el negocio y encaminarlo hacia la consecución de los objetivos de la entidad, en todas las categorías: operaciones, información financiera y cumplimiento. El talento humano que trabaja en una organización debe no solo asimilar la información, sino también compartirla en virtud de que este factor destaca la importancia y la necesidad de mantener canales permanentes de intercambio de información que permitan la transmisión eficaz del conocimiento de cada miembro de la organización.

(Mantilla Blanco, 2005), señala que cada empresa debe capturar información pertinente, financiera y no financiera, relacionada con actividades o eventos tanto externos como internos. Es necesario reconocer que los sistemas de información puede ser formales o informales y pueden obtenerse mediante cuestionarios, entrevistas, estudios de la demanda del mercado, entre otros. Los sistemas de información deben cambiar de acuerdo con las necesidades para apoyar los nuevos objetivos de la entidad relacionados.

\subsubsection{Monitoreo}

Con la finalidad de alcanzar el concepto de mejoramiento continuo, todo proceso que se desarrolle en una organización debe ser supervisado y monitoreado; para ello se requiere de un sistema de control interno flexible que responda de manera ágil y se adapte a las condiciones.

Al monitoreo se lo clasifica en permanente o periódico en relación a su periodicidad. El monitoreo será periódico cuando existan plazos establecidos para su desarrollo, en tanto que el monitoreo es permanente cuando se realizan de forma continúa presentándose de manera jerarquizada, es decir, se revisan los niveles inferiores hasta llegar a los niveles superiores, quienes son los responsables de realizarlo. 
Por lo tanto, los sistemas de control interno requieren que sean monitoreados, valorando el desempeño del sistema en el tiempo, o sea, en el curso de las operaciones. El alcance y la frecuencia de las evaluaciones separadas dependerán de la valoración de riesgos y de la efectividad de los procedimientos de supervisión empleados.

\subsection{Métodos para Evaluar el Control Interno}

La evaluación del Sistema de Control Interno de cualquier organización, independientemente de su naturaleza económica y social, tiene como finalidad valorar la situación actual de sus operaciones a fin de establecer acciones correctivas a partir de los resultados alcanzados. Todo aquello se desarrolla en varios momentos de control, pudiendo ser: previo, continuo o posterior al ejercicio de las operaciones sujetas a evaluación. Esta actividad evalúa los riesgos presentes y ofrece instrumentos de análisis para mitigarlos en los procesos, de no ser así, se establecerían alternativas para efectuar un mejoramiento continuo del sistema.

Por lo tanto, en búsqueda de la teoría de la calidad total, al aplicar un sistema de control interno se hace necesario para la valoración permanente del funcionamiento del mismo, varias herramientas que beneficiarán a la organización y a los miembros, entre ellos se presentan: cuestionarios, flujogramas, matrices, cédulas narrativas, entre otros.

\subsubsection{Método de Cuestionario o Preguntas para Evaluar el Sistema de Control Interno}

Esta herramienta se diseña en base a preguntas dirigidas a los responsables de los departamentos, áreas, procesos o componentes bajo examen. Con las respuestas obtenidas, se analizará la existencia de controles funcionales, que luego permitirá al auditor desarrollar procedimientos de cumplimiento para garantizar la funcionalidad del sistema de control interno implementado. Esta herramienta valora de forma cuantitativa la funcionalidad de los controles, dando como respuesta un nivel de confianza y riesgo en las operaciones.

\subsubsection{Método Narrativo o de Prosa para Evaluar el Siste- ma de Control Interno}

Comprende una descripción escrita que prepara el auditor en observancia a la valoración de los controles inspeccionados en las áreas sujetas a examen, esta evaluación integra las declaraciones realizadas a los funcionarios responsables de los procesos, mediante la aplicación de entrevistas, encuestas y diálogos. Este instrumento valora de forma cualitativa la estructura, características y procedimientos integrados al sistema de control interno de la organización y establece recomendaciones para la mitigación de los riesgos presentes.

\subsubsection{Método Gráfico para Evaluar el Sistema de Control Interno}

Consiste en la representación de los controles presentes en las áreas sujetas a evaluación mediante el empleo de flujogramas, gráficas y símbolos capaces de describir la estructura orgánica de los procedimientos analizados. En observancia a los objetivos de la entidad se desarrollan los procedimientos y a la par se presentan los riesgos que podrían afectar en los resultados.

Argumentada la investigación con los conceptos antes presentados, se deriva el objetivo general de este trabajo, que consiste en: evaluar comparativamente el sistema del control interno del sector privado y del sector público del cantón Morona.

Los resultados alcanzados permitirán fortalecer los sistemas de control interno, que promoverá la mejora continua de las organizaciones inmersas en el caso de estudio, contribuyendo a establecer elementos generales para un desempeño eficiente en la administración y gestión de las entidades públicas y privadas.

\section{Metodología}

Esta investigación de tipo explicativa, presenta de modo sistemático las características de una población, situación o área de interés, en este caso el sistema de control interno de las entidades públicas y privadas. Se desarrolla con un enfoque cuantitativo, debido a la valoración numérica que se presenta en la calificación de los niveles de riesgo y confianza en cada uno de los componentes del Sistema de Control Interno, según COSO I, fundamentada en revisión bibliográfica y análisis de fuentes secundarias que abordan el tema del control interno, presentados en textos escritos y consultas artículos de bases científicas mediante internet.

Esta investigación se llevó a cabo en el cantón Morona, provincia de Morona Santiago, en el Ecuador, tomando como universo, a 500 instituciones comerciales y 33 instituciones públicas; siendo la muestra de 341 instituciones privadas y 33 públicas. Se toma todo el universo del sector público, debido a la cantidad minúscula de las empresas.

Para la recolección de la información se realizaron entrevistas con los responsables y administradores de las diferentes instituciones públicas y privadas. La herramienta básica para la evaluación de los sistemas de control interno ha sido el Cuestionario de Control Interno, el mismo que fue elaborado en observancia al cumplimiento del Modelo de Control COSO I.

Los componentes de control interno según el modelo de control de esta investigación, pretende evaluar el cumplimiento de las empresas a los requerimientos presentados en cada componente según COSO I. En el sector público, los componentes del modelo, se encuentran traducidos a Normas Técnicas de Control Interno, cuyo cumplimiento es obligatorio. Sin embargo en el sector privado, en los requerimientos de control según COSO, se evidencia en el desarrollo de las operaciones, sin disponer de documentación. 
La Tabulación de los resultados nos permitió determinar el nivel de riesgo y confianza de los cinco componentes evaluados en las instituciones para realizar la comparación valorando el nivel de confianza y el nivel de riesgo por cada variable de cada componente.

Donde el nivel de confianza, constituye los resultados de la funcionalidad de los controles integrantes del sistema de control interno, en cambio el nivel de riesgo, se define como la inobservancia a las disposiciones de control o la carencia de las mismas, en el área, proceso o entidad sujeta a evaluación.

\section{Resultados}

De los resultados obtenidos con la aplicación del cuestionario de control interno, tanto al sector público como al privado, se presentan las siguientes valoraciones comparativas de los cinco componentes sujetos a evaluación, (Fig. 1).

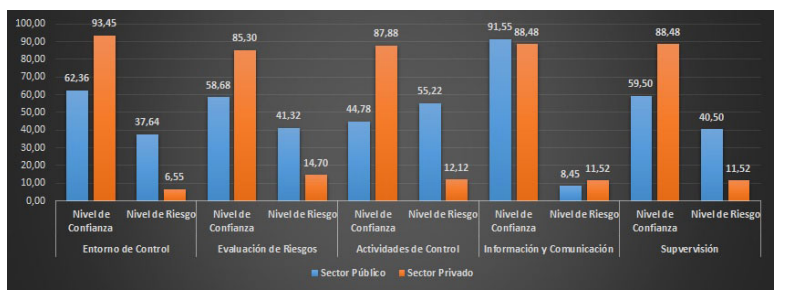

Figura 1. Comparación entre los componentes de COSO I. Fuente: Datos obtenidos en la aplicación del cuestionario de la investigación elaborado por las autoras

Las operaciones y las actividades empleadas en el desarrollo administrativo y económico de una entidad, apoyadas en el marco de normas e instrumentos de valoración continua, permiten realimentar el ciclo de operaciones convirtiendo al Sistema de Control Interno en un conjunto armónico, que involucra el compromiso de todos los niveles de la organización en el cumplimiento de control: la gerencia mediante el conocimiento general y la evolución de resultados; el diseño de procedimientos y normas para el desarrollo de actividades por parte de áreas administrativas, a través del uso apropiado de procesos administrativos como son la planeación, evaluación del desempeño de personal y el control de gestión de la empresa u organización.

El Sistema de Control Interno se integra por procedimientos, métodos y actividades diseñadas en específico para cada operación, bajo el marco presentado por la filosofía de la dirección, cuyo propósito es promover una seguridad razonable en los procedimientos efectuados para alcanzar los objetivos declarados por la administración.

\subsection{Entorno de Control}

Al evaluar el primer componente de control Interno, actividades de control, comparativamente entre el sector público y el sector privado, (Fig. 2), se demuestra que el diseño organizacional, la autoridad asignada, la responsabilidad asumida, la gestión de talento humano, los valores

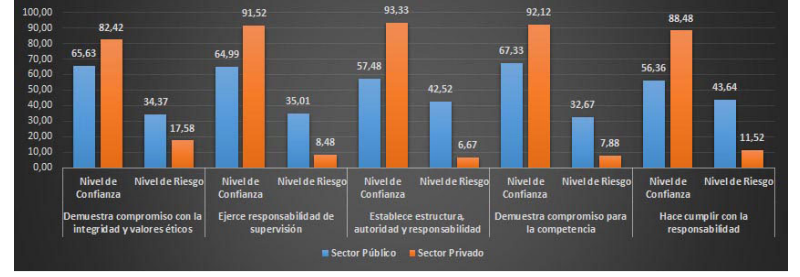

Figura 2. Evaluación comparativa componente Entorno de control. Fuente: Datos obtenidos en la aplicación del cuestionario de la investigación elaborado por las autoras

éticos y la integridad, se abordan de forma prioritaria en el sector público con relación al sector privado.

Esto debido a los marcos legales previstos para el efecto, tales como: principios y valores declarados, códigos de ética, leyes generales, reglamentos internos, instructivos y demás disposiciones de cumplimiento obligatorio en todas las operaciones.

Al caracterizar el componente ambiente de control en el sector privado, se presenta un alto compromiso de los miembros de la organización en el desarrollo de sus operaciones enmarcadas por la integridad y valores éticos de cumplimiento con la filosofía de la alta gerencia. De forma tal, que todas las operaciones son supervisadas de manera constante.

Se considera que en el sector público y privado, la dirección demuestra interés por conseguir, desarrollar y retener colaboradores comprometidos con los objetivos de la organización. Para aquello, se apoyan en la instrumentalización de políticas internas declaradas o presentadas como prácticas generales desarrolladas por la entidad con el afán de constituir una guía que integra los requerimientos y expectativas de los accionistas e inversionistas.

Estos elementos promueven la identificación de competencias necesarias en la organización y de las bases legales para el ejercicio de sus cargos, de forma simultánea al análisis de los resultados que van siendo alcanzados se establecen las medidas correctivas que redefinan su ejercicio.

\subsection{Evaluación de Riesgos}

En la valoración del componente evaluación de riesgos (Fig. 3), estudiado en el Sector Público y Privado, se conocen variadas alternativas e instrumentos elaborados para prevenir, mitigar, analizar y reducir los riesgos identificados en el entorno de la organización. En el Sector Público, como resultado de una evaluación interna se identifican, valoran y cuantifican los riesgos potenciales, sean internos y/o externos que puedan afectar al cumplimiento de metas, posterior a aquello la máxima autoridad y los responsables de los procesos, diseñan instrumentos de planificación tales como: planes de mitigación, matrices de riesgos, para definir las estrategias que permitan reducir los impactos o evitar los riesgos. 
En el análisis de las operaciones y las alternativas a desarrollar se valora el costo-beneficio de los procedimientos a implementar.

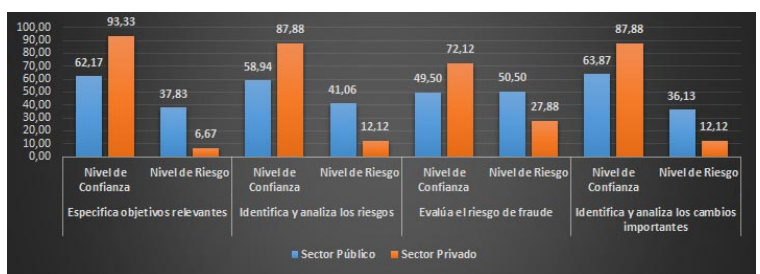

Figura 3. Comparación del componente Evaluación de riesgo. Fuente: Datos obtenidos en la aplicación del cuestionario de la investigación elaborado por las autoras

A través del estudio de la muestra considerada del sector público, se reconoce que a pesar de no elaborar ni emplear instrumentos de planificación definidos: planes operativos, estratégicos, presupuestos, matrices para el control de riesgos, entre otros. La entidad identifica riesgos a través de la comunicación interna con todos los miembros, y establece acciones correctivas en alcance a sus recursos.

Las deficiencias encontradas en el sector privado obedecen a: la carencia de documentar los procesos, la ausencia de evidencias escritas que demuestren las estrategias desarrolladas para evitar, mitigar y administrar los riesgos.

Frente a los cambios que podrían afectar a la consecución de los objetivos en una organización, la administración establecer las funciones y responsabilidad de todo el personal para la administración de los potenciales riesgos. Para esto se adaptan tareas, estructuras y procesos tecnológicos limitando el impacto de los resultados presentes y evitando nuevos riegos.

\subsection{Actividades de Control}

Al evaluar el componente, actividades de control (Fig. 4), integrado por: controles en los sistemas de información y las principales actividades de control para el desempeño de sus operaciones, se presenta de forma ordenada, sistemática y procedimentado el empleo de los recursos tecnológicos de la información en el sector público, mientras que en el sector privado existen limitaciones al conseguir que las tecnologías de información y comunicación respondan a documentos previamente diseñados, lo cual limita la evaluación del funcionamiento.

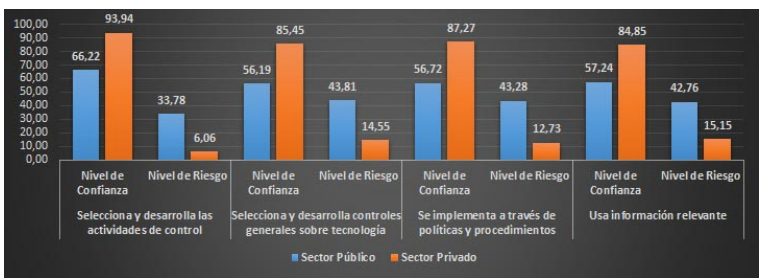

Figura 4. Evaluación comparativa componente Actividades de Control. Fuente: Datos obtenidos en la aplicación del cuestionario de la investigación elaborado por las autoras
En el sector público la gestión de estos recursos se realiza a través de procesos de tecnología de información, a través de acciones de control mediante la implementación de lineamientos, que establece los resultados planificados y los procedimientos a emplear para hacerlos efectivos.

En tanto que, en el sector privado, las acciones de control se diseñan e implementan en concordancia al estilo administrativo en base a sus recursos. Con la consideración de que la costumbre ha sido el principio fundamental para el cumplimiento en las entidades del sector privado.

\subsection{Información y Comunicación}

El componente información es considerado como un factor que enfatiza la importancia de la comunicación que se produce por agentes externos e internos.

Como consecuencia de lo expuesto, en el componente información y comunicación (Fig. 5), se demuestra que en el sector público, se emplean sistemas informáticos para la elaboración de los informes de actividades, en respuesta a los planes que podrían ser: operativos, financieros, de administración, entre otros. Siendo observado el cumplimiento de las normas que permite dirigir y controlar las operaciones de manera adecuada.

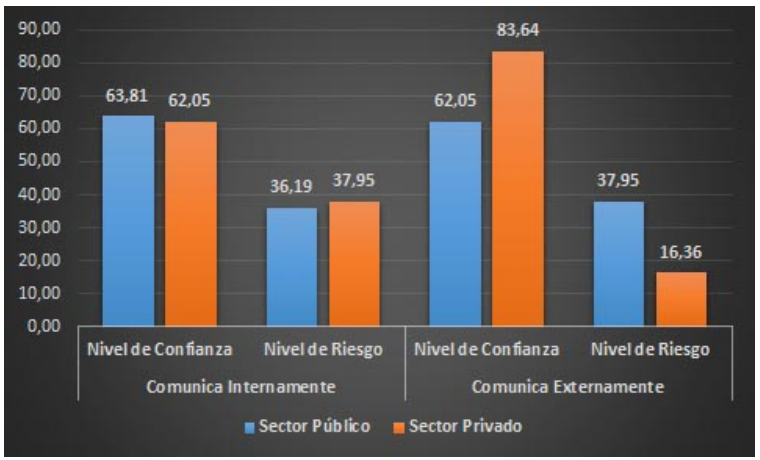

Figura 5. Evaluación comparativa componente Información y Comunicación. Fuente: Datos obtenidos en la aplicación del cuestionario de la investigación elaborado por las autoras

En tanto, que en el sector privado, los sistemas de información y comunicación se sustentan en softwares contables, y comunicaciones directas con los empleados, clientes y proveedores.

\subsection{Supervisión o Monitoreo}

Constituye un proceso que controla el desempeño de todos los elementos presentes en los componentes antes detallados en función al tiempo, es decir, evalúan la calidad del funcionamiento del sistema de control interno, a fin de retroalimentar las acciones implementadas o modificar.

En el sector público, (Fig. 6) las actividades de monitoreo incluyen actividades de control y revisión permanente, por medio de las unidades de auditoría interna, o por la dirección de cada organización, mientras que en el sector privado la supervisión la realiza el gerente o administrador 
a través de la observación a las actividades desarrolladas por el personal, cuya retroalimentación la efectúa de forma verbal y en el momento que ocurren los hechos, no se llevan registros históricos ni documentados.

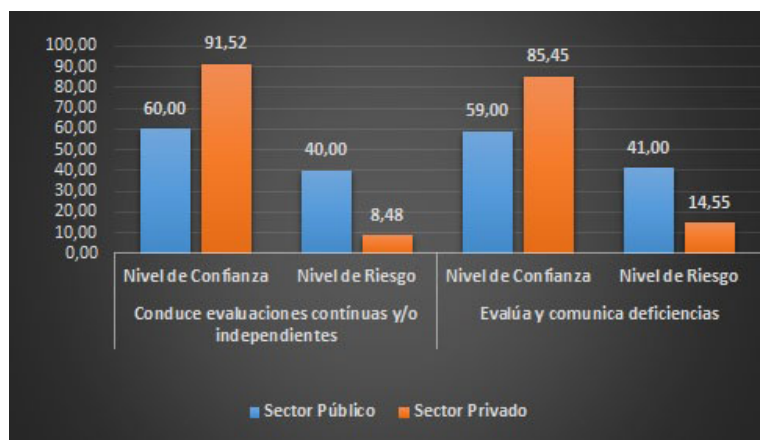

Figura 6. Evaluación comparativa componente Supervisión. Fuente: Datos obtenidos en la aplicación del cuestionario de la investigación elaborado por las autoras

\section{Conclusiones}

Investigar el contenido "Control Interno" según el modelo internacional COSO, demuestra varios aportes de conocimiento y aplicación, en este caso, el interés ha sido el análisis de este marco conceptual en el sector público y privado del Cantón Morona.

En la muestra de estudio, establecida en 33 casos de estudio en el sector privado y 341 casos en el sector público, revela en las organizaciones un alto compromiso hacia el cumplimiento de los objetivos de la organización y el desempeño empresarial deseado. Sin embargo, los controles y procedimientos diseñados para cada área se diferencian de acuerdo a la normativa legal del sector investigado.

A partir de los resultados de la investigación se puede universalizar que en el sector Público, las actividades y procedimientos dirigidos hacia el control interno, se definen en marcos legales de aplicación obligatoria, presentados a través de: normas internas, reglamentos, manuales, disposiciones, entre otros, cuya inobservancia acarrea una responsabilidad administrativa, por parte del órgano de control público, la Contraloría General del Estado.

En tanto que el Sector Privado, debido a las limitaciones en el diseño de herramientas de planificación y control enmarcadas en un estudio documental, las operaciones se realizan en cumplimiento a órdenes verbales o a la costumbre, sin embargo, el estilo de la administración, promueve el cumplimiento de valores éticos en el ejercicio de las funciones.

En consideración a que el Sistema de Control Interno comprende un criterio fundamental para la consecución de los objetivos, los fundamentos se basan en sistemas y estudios necesarios para enmarcar las actividades administrativas, financieras y operativas bajo estándares que garantizan la salvaguarda de activos, implementados en basa a un análisis de la relación costo-beneficio. En esencia, el costo del Sistema de Control Interno se debe diferenciar por el grado de mitigación de riesgos y el impacto que alcanza su aplicación.

El reto es encontrar el justo equilibrio en el diseño del Sistema de Control Interno; un excesivo control puede ser costoso y contraproducente. Es necesario reconocer que un sistema de control interno diseñado de acuerdo a las necesidades de la organización, constituye una garantía para la consecución de los objetivos en una entidad, siendo este elemento continuamente revisado y actualizado a las demandas de la organización.

\section{Referencias}

Blanco Luna, Y. (2012). Auditoría integral normas y procedimientos. Bogotá: Ecoe Ediciones, Bogota, Colombia., 2da edición.

Mantilla Blanco, Samuel Alberto; Cante S., S. Y. (2005). Auditoría del Control interno. Ecoe Ediciones, Bogotá.

Whittington, R y Pany, K. (2005). Principios de auditoría. McGraw-Hill Interamericana, Bogotá.

Rivas Márquez, G. (2011). Modelos contemporáneos de control interno. fundamentos teóricos. Observatorio Laboral Revista Venezolana, 4(8):115-136.

Viloria, N. (2005). Factores que inciden en el sistema de control interno de una organización. Actualidad Contable Faces, 8(11):87-92.

Coopers, y Lybrand,. (1997). Los nuevos conceptos de control interno. (Informe COSO). Ediciones Díaz Santos, España, 1ra edición.

Recibido: 01 de junio de 2016

Aceptado: 28 de abril de 2017

López Jara, Ana Alexandra: Ingeniera en Contabilidad y Auditoría; M Sc. en Auditoría Integral, Profesora Titular de la Carrera de Contabilidad y Auditoría, Universidad Católica de Cuenca Sede Macas.

Pesántez Rodríguez, Judith Cristina: Contadora Pública Auditora; M Sc. en Contabilidad y Finanzas, Profesora Titular de la Carrera de Contabilidad y Auditoría, Universidad Católica de Cuenca Sede Macas. 
